\title{
NEUROMUSCULAR ATTRIBUTES ARE ASSOCIATED WITH POOR MOBILITY IN OLDER ADULTS WITH VASCULAR RISK CONDITIONS
}

\author{
A.J. JOR'DAN ${ }^{1,2}$, M.E. JACOB ${ }^{1,3,4,5}$,E. LERITZ $Z^{1,2}$, J.F. BEAN ${ }^{1,3,5}$ \\ 1. New England Geriatric Research, Education, and Clinical Center, VA Boston Healthcare System, Boston, MA, USA; 2. Department of Psychiatry, and 3. Department of Physical

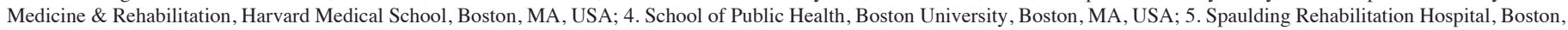 \\ MA, USA.

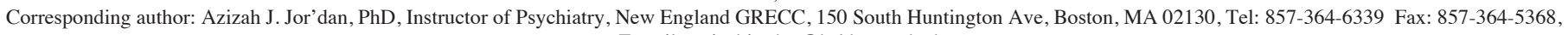 \\ E-mail: azizahjordan@hsl.harvard.edu
}

\begin{abstract}
Background: The mobility of older adults is limited by the compounding effects of vascular health conditions, or vascular risk burden. However, little is known about the role of neuromuscular attributes among those in which vascular risk burden contributes to mobility limitations. Objective: We investigated (1) the relationship between the absence/presence of type 2 diabetes, hypertension, and/or obesity and mobility measures and neuromuscular attributes, and (2) whether the association between vascular risk burden and mobility is mediated by lower limb neuromuscular attributes. Design: Cross-sectional analysis of baseline data from 430 older adults within the Boston RISE Study. Measurements: Measures of mobility were the Short Physical Performance Battery, habitual gait speed, and functional mobility as measured by the Late Life Function Instrument. We also evaluated lower limb neuromuscular attributes, namely leg strength, leg velocity, trunk extensor muscle endurance, knee and ankle range of motion, and sensory loss. Results: Participants selfreported the presence of None $(n=93)$, One $(n=179)$, Two $(n=114)$, or Three $(n=44)$ of the following conditions: diabetes, hypertension, and obesity. Multivariable regression models indicated that those with a greater vascular risk burden had worse performance on the Short Physical Performance Battery $(\mathrm{p}=0.01)$, slower gait speed $(p=0.0003)$ and lower Basic and Advanced Late Life Function Instrument scores $(p<0.003)$. These associations were independent of multiple covariates. Vascular risk burden was also found to be negatively associated with leg strength $(\mathrm{p}=0.0002)$ and knee flexion range of motion $(\mathrm{p}<0.0001)$ and an associated non-significant trend was observed with leg velocity $(\mathrm{p}=0.06)$. In addition, the association between vascular risk burden and mobility outcomes were found to be partially mediated by leg strength, leg velocity, and knee flexion range of motion. Conclusions: Among older adults with vascular risk burden and mobility problems, neuromuscular impairments in attributes such as leg strength, leg velocity, and knee range of motion may need to be treatment priorities.
\end{abstract}

Key words: Vascular risk, mobility, neuromuscular attributes, cardiovascular disease.

\section{Introduction}

Mobility is important in the lives of older adults as it helps to maintain independence. The decline in mobility has been separately linked to vascular risk factors such as obesity, hyperglycemia, hyperlipidemia, and hypertension in older adults (1-2). For example, diabetes and hypertension are independently linked to slow gait speed, a metric of mobility and a vital indicator of mortality (1). An increasing number of vascular risk components (i.e., hypertension, high C-reactive protein, obesity, diabetes, and smoking) has been shown to linearly increase the likelihood of gait speed decline, and disability among older adults (2). In parallel, other studies have independently linked poor mobility outcomes to lower limb neuromuscular attributes, such as leg strength and velocity, trunk muscle endurance, and range of motion (ROM) among older adults (3). There is also evidence suggesting that Metabolic Syndrome (i.e., the presence of three or more of the following: hypertension, hyperglycemia, hyperlipidemia, and abdominal obesity), is associated with lower limb weakness among men (4). However, it is not known if the compounding Received March 31, 2019 Accepted for publication July 1, 2019 of vascular risk, or vascular risk burden (VRB), affects other lower limb neuromuscular attributes.

Vascular risk factors rarely occur in isolation (5), and it is not evident how the co-existence of multiple vascular conditions, or the VRB, influences mobility in older adults. Additionally, the mechanisms by which VRB influence mobility are not clear.

Therefore, the aim of this study was to determine the link between VRB and 1) mobility as assessed by the Short Physical Performance Battery (SPPB), habitual gait speed, and the Late Life Function Instrument (LLFI); and 2) lower limb neuromuscular attributes (e.g., leg strength and leg velocity) and to determine whether the potential associations between VRB and mobility measures were mediated by lower limb neuromuscular attributes. We hypothesized that older adults with a greater VRB will have poorer mobility performance and be deficient in lower limb neuromuscular attributes. We further hypothesized that lower limb neuromuscular factors that are associated with VRB status, will mediate the association between VRB and mobility. 


\section{THE JOURNAL OF FRAILTY \& AGING}

\section{Methods}

\section{Study Design}

This cross-sectional study used the data from the 430 community-dwelling primary care patients who participated in the Boston Rehabilitative Impairment Study of the Elderly (Boston RISE) and were examined at baseline between December 2009 and January 2012. This prospective cohort study aimed to identify modifiable neuromuscular impairments that are associated with mobility decline in older adults. The design and methodology for this study has been previously published in detail (6). Briefly, to be included, individuals had to be $\geq 65$ years of age, able to understand and communicate in English, and have difficulty or task modification with walking half a mile or climbing one flight of stairs. The exclusion criteria were: 1) presence of a terminal disease (e.g. receiving hospice services, metastatic cancer), 2) major surgery or myocardial infarction in the last 6 months, 3) planned major surgery, 4) planned move from the area within 2 years, 5) MiniMental Status Exam (MMSE) score <18, or 6) major medical problems that interfered with safe and successful testing (e.g., history hip replacement with recurrent dislocation, uncontrolled hypertension, loss of lower extremity). All participants provided written informed consent as approved by the Institutional Review Board of Spaulding Rehabilitation Hospital.

\section{Data Collection}

Socio-demographic characteristics (age, sex, race, education) and disease status (type 2 diabetes and hypertension) were selfreported using a comorbidity questionnaire that consisted of 17 frequent general practice conditions for which patients were receiving treatment and/or had limitations on their activities (7). Study staff measured height and weight using standardized techniques, calculated BMI, and classified participants as obese if their BMI was 30 or higher. Depression severity was assessed using the Patient Health Questionnaire-9 (PHQ-9), and cognitive ability was assessed using the MMSE.

\section{Mobility Measures}

Mobility measures consisted of the following: 1) SPPB, a reliable and valid screening test used to characterize lower extremity function (8). The test includes measures of progressive standing balance, habitual walking speed, and chair stand ability; 2) gait speed, a sensitive measure for assessing functional status and overall health. This measure was collected during two 4-meter usual pace walking trials from the SPPB; and 3) the Basic and Advanced Lower Limb Function subdomains of the LLFI, a validated questionnaire that captures the participant's perceived ability to do specific aspects of their daily routines (function) and is a component of the larger Late Life Function and Disability Instrument (9). Given the aim of the current study, which focused on mobility outcomes, we evaluated scores on the Basic and Advanced lower extremity function subdomains of the LLFI.

\section{Neuromuscular Measures}

Leg strength was measured by determining the 1 repetition maximum (1RM) for each leg with a Keiser pneumatic leg press machine using a previously published protocol (10). Peak leg strength was defined as the maximum value observed on either side. Peak power was defined as the highest recorded power out of five trials performed with each leg at $40 \%$ and $70 \%$ of the 1RM. Peak leg velocity was calculated by dividing peak power by the graphically displayed force at peak power recorded during the testing. Trunk extensor muscle endurance (11) was measured as the length of time in seconds (up to 150 seconds) that the participant was able to maintain their trunk in a neutral position within the sagittal plane in line with their pelvis and legs with arms crossed against the chest. Knee and ankle ROM were measured with a goniometer (12). Ankle ROM was considered to be impaired if there was inability to dorsiflex past $90^{\circ}$ or plantar flex past $110^{\circ}$ in either leg. Foot sensation was measured over the dorsum of the big toes using the Semmes-Weinstein monofilament test. Both impaired ankle ROM and sensory loss were dichotomized as being present or absent.

\section{Statistical Analysis}

Participants were classified into VRB groups (i.e., None, One, Two or Three condition[s]) based on the self-reported absence or presence of type 2 diabetes, hypertension, and obesity. VRB groups were characterized using means and standard deviations for continuous variables and frequency (percentage) for categorical data. The Kruskal-Wallis test was used to determine if the group demographics, PHQ-9, MMSE, mobility measures, and the neuromuscular attributes differed by VRB.

Multivariable regression analyses were used to examine the association between VRB (predictor) and 1) mobility measures (i.e., SPPB total score, gait speed, Basic and Advanced LLFI), and 2) neuromuscular attributes (leg strength, leg velocity, trunk extensor muscle endurance, knee flexion ROM, ankle ROM, and sensory loss). Separate models were developed for each outcome and were adjusted for the pre-specified confounders of age, race, sex, PHQ-9, and MMSE scores. Mediation analysis using regression was subsequently conducted to determine whether the potential associations between VRB and mobility measures were in part mediated by neuromuscular attributes. Attributes that demonstrated 1) a significant association with VRB or 2) a clinically meaningful group difference, as determined by previous literature, were introduced into mobility measure regression models. A mediator effect was defined as a reduction in the estimate by $>10 \%$ when the neuromuscular attribute was included in the regression model $(13,14)$. Significance levels were set to $\mathrm{p}<0.05$. All analyses were performed using JMP software (SAS Institute, Cary, NC). 
Table 1

Complete population demographic and clinical characteristics

\begin{tabular}{|c|c|c|c|c|c|}
\hline & \multicolumn{4}{|c|}{ VRB } & \multirow[b]{2}{*}{$\mathbf{P}$} \\
\hline & None $\mathbf{N}=93$ & One $N=179$ & Two N=114 & Three N=44 & \\
\hline Age, years & $77.4(6.8)$ & $78.0(7.2)$ & $75.3(6.7)$ & $72.3(4.9)$ & $<0.001 *$ \\
\hline Female, $\%$ & 65.6 & 68.2 & 65.8 & 75.0 & 0.69 \\
\hline Non-white, $\%$ & 11.8 & 17.3 & 17.5 & 29.6 & 0.09 \\
\hline BMI, kg/m2 & $25.6(2.8)$ & $27.3(4.5)$ & $33.6(6.4)$ & $36.2(5.4)$ & $<0.001 *$ \\
\hline Education, $\%<\mathrm{HS}$ & 14.0 & 11.2 & 13.2 & 13.6 & 0.14 \\
\hline PHQ-9 & $1.5(3.9)$ & $1.3(3.0)$ & $1.1(2.4)$ & $1.6(3.4)$ & 0.88 \\
\hline Mini-mental State Exam & $27.5(2.3)$ & $27.5(2.2)$ & $27.2(2.5)$ & $27.4(2.7)$ & 0.68 \\
\hline \multicolumn{6}{|l|}{ Mobility Measures } \\
\hline SPPB Total & $8.8(2.4)$ & $8.7(2.3)$ & $8.8(2.1)$ & $8.0(2.1)$ & 0.14 \\
\hline Gait Speed, m/s & $0.94(0.2)$ & $0.90(0.2)$ & $0.91(0.2)$ & $0.83(0.2)$ & $0.02 *$ \\
\hline Basic LLFI & $66.9(12.7)$ & $66.6(12.2)$ & $67.0(11.9)$ & $59.5(9.6)$ & $<0.001 *$ \\
\hline Advanced LLFI & $44.3(13.0)$ & $43.8(14.9)$ & $40.1(12.5)$ & $32.7(18.5)$ & $<0.001 *$ \\
\hline \multicolumn{6}{|l|}{ Neuromuscular Attributes } \\
\hline Leg Strength, kg & $9.7(2.3)$ & $9.7(2.7)$ & $9.3(2.4)$ & $8.2(2.2)$ & $0.002 *$ \\
\hline Leg Velocity, m/s & $0.99(0.2)$ & $1.01(0.2)$ & $1.01(0.3)$ & $0.93(0.3)$ & 0.34 \\
\hline Trunk Extension Endurance, s & $100.1(55.5)$ & $95.6(58.7)$ & $93.9(60.5)$ & $88.2(61.1)$ & 0.73 \\
\hline Knee Flexion, deg & $128.6(9.8)$ & $127.4(12.5)$ & $120.0(14.8)$ & $116.8(15.5)$ & $<0.001^{*}$ \\
\hline Ankle ROM Impairment, $\%$ yes & 23.7 & 32.0 & 23.7 & 36.4 & 0.19 \\
\hline Sensory Loss, $\%$ yes & 33.7 & 26.3 & 27.7 & 34.9 & 0.49 \\
\hline
\end{tabular}

\section{Results}

Group demographics, mobility, and neuromuscular characteristics are listed in Table 1 . Those with no vascular conditions represented $22 \%$ of the cohort, $42 \%$ had one vascular condition, $26 \%$ had two vascular conditions, and $10 \%$ had three vascular conditions. The groups were similar in sex, race, education, and MMSE scores. Gait speed, Basic and Advanced LLFI, lower leg strength, and knee flexion ROM differed by VRB group.

Regression analysis determined that VRB status was linked to performance on the SPPB $(\mathrm{p}=0.01)$, gait speed $(\mathrm{p}=0.0003)$, and lower Basic $(\mathrm{p}=0.003)$ and Advanced LLFI scores $(\mathrm{p}<0.0001)$ (Table 1), such that those with a greater VRB had worse performance on these mobility measures, after adjusting for age, race, sex, PHQ-9, and MMSE scores (Table 3, Model $1)$.

As shown in Table 2, those with a greater VRB were shown to have higher impairment in lower leg strength $(\mathrm{p}=0.0002)$ and knee flexion ROM ( $\mathrm{p}<0.0001)$, independent of age, race, sex, PHQ-9, and MMSE scores. Of note, although statistically significant, the variation specifically explained by the knee flexion model is weak. Leg velocity did not achieve statistical significance $(\mathrm{p}=0.06)$, however, the observed differences between groups $(0.09 \mathrm{~m} / \mathrm{s})$ exceeded what is defined as a clinically meaningful threshold in the literature (15) and leg velocity was therefore included in subsequent mediation analyses. Trunk extension muscle endurance, ankle ROM, or sensory loss did not differ by VRB status (Table 2).

Regression analysis was used to test whether VRB status predicted mobility (i.e., SPPB, gait speed, Basic and Advanced LLFI) when significant and clinically meaningful lower limb neuromuscular attributes (i.e., leg strength, knee flexion, and leg velocity) were included as potential mediators. As shown in Table 3, when lower leg strength was included in the subsequent models (Model 2), VRB was no longer associated with SPPB ( $\mathrm{p}=0.49$ ) or Basic LLFI ( $\mathrm{p}=0.07)$. When leg strength was added to the model, VRB coefficients were attenuated by 


\section{THE JOURNAL OF FRAILTY \& AGING}

Table 2

Separate Univariate and Multivariable Regression Models Evaluating the Association between Vascular Risk Burden (VRB) and Measures of Mobility and Neuromuscular Attributes

\begin{tabular}{|c|c|c|c|c|c|c|c|c|c|}
\hline & $\begin{array}{c}\text { Model } \\
\mathbf{R}^{2} \\
\end{array}$ & $\begin{array}{c}\text { Statistics }^{\mathrm{a}} \\
\mathbf{P}\end{array}$ & $\begin{array}{c}\text { Model } \\
\mathbf{R}^{2} \\
\end{array}$ & $\begin{array}{c}\text { Statistics }^{\mathrm{b}} \\
\text { P }\end{array}$ & None $\mathbf{N}=\mathbf{9 3}$ & One $N=179$ & $\begin{array}{c}\text { VRB } \\
\text { Two N=114 }\end{array}$ & Three N=44 & $\mathbf{P}$ \\
\hline \multicolumn{10}{|l|}{ OUTCOMES } \\
\hline \multicolumn{10}{|l|}{ Mobility Measures } \\
\hline SPPB Total & 0.01 & 0.218 & 0.14 & $<0.0001$ & $8.9 \pm 0.2$ & $8.8 \pm 0.2$ & $8.7 \pm 0.2$ & $7.7 \pm 0.3$ & $0.01 *$ \\
\hline Gait Speed, m/s & 0.02 & 0.038 & 0.23 & $<0.0001$ & $0.96 \pm 0.2$ & $0.92 \pm 0.1$ & $0.90 \pm 0.02$ & $0.80 \pm 0.03$ & $0.0003 *$ \\
\hline Basic LLFI & 0.03 & 0.003 & 0.11 & $<0.0001$ & $67.5 \pm 1.2$ & $67.4 \pm 0.9$ & $67.4 \pm 1.1$ & $60.3 \pm 1.8$ & $0.003^{*}$ \\
\hline Advanced LLFI & 0.06 & $<0.0001$ & 0.16 & $<0.0001$ & $45.8 \pm 1.4$ & $45.5 \pm 1.1$ & $40.9 \pm 1.3$ & $33.1 \pm 2.1$ & $<0.0001 *$ \\
\hline \multicolumn{10}{|l|}{ Neuromuscular Attributes } \\
\hline Leg Strength, kg & 0.03 & 0.005 & 0.21 & $<0.0001$ & $10.1 \pm 0.2$ & $10.2 \pm 0.2$ & $9.5 \pm 0.2$ & $8.4 \pm 0.4$ & $0.0002 *$ \\
\hline Leg Velocity, m/s & 0.009 & 0.358 & 0.29 & $<0.0001$ & $1.04 \pm 0.02$ & $1.06 \pm 0.02$ & $1.04 \pm 0.02$ & $0.95 \pm 0.04 \dagger$ & 0.06 \\
\hline Trunk Extension Endurance, $\mathrm{s}$ & 0.003 & 0.742 & 0.06 & 0.0018 & $102.6 \pm 6.2$ & $98.8 \pm 4.6$ & $94.2 \pm 5.7$ & $85.2 \pm 9.4$ & 0.43 \\
\hline Knee Flexion, deg & 0.10 & $<0.0001$ & 0.12 & $<0.0001$ & $128.9 \pm 1.4$ & $127.9 \pm 1.0$ & $120.0 \pm 1.2$ & $116.2 \pm 2.0$ & $<0.0001 *$ \\
\hline Ankle ROM Impairment, $\%$ yes $^{c}$ & 0.01 & 0.189 & 0.03 & 0.0847 & $24.6 \pm 0.05$ & $32.8 \pm 0.04$ & $25.7 \pm 0.04$ & $40.8 \pm 0.070$ & 0.14 \\
\hline Sensory Loss, $\%$ yes $^{\mathrm{c}}$ & 0.006 & 0.493 & 0.10 & $<0.0001$ & $34.6 \pm 0.05$ & $28.2 \pm 0.03$ & $32.2 \pm 0.04$ & $46.3 \pm 0.07$ & 0.12 \\
\hline
\end{tabular}

Model Statistics ${ }^{\mathrm{a}}$ - Univariate $\mathrm{R}^{2}$ and p-value for the overall regression model; Model Statistics ${ }^{\mathrm{b}}-$ Multivariable $\mathrm{R}^{2}$ and $\mathrm{p}$-value for the overall regression model; VRB - Data $=$ Least Square

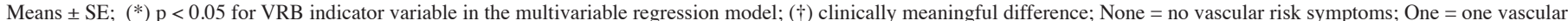

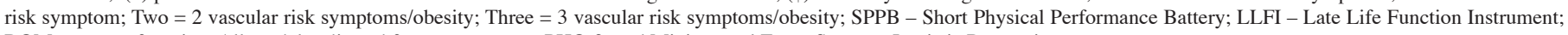
ROM - range of motion; All models adjusted for age, race, sex, PHQ-9, and Mini-mental Exam Scores; ${ }^{\mathrm{c}}$ Logistic Regression

\section{Table 3}

Separate Multivariable Regression Models Examining Mediation of the Association between Vascular Risk Burden (VRB) and Mobility by Leg Strength, Knee Flexion ROM, or Leg Velocity

\begin{tabular}{|c|c|c|c|c|c|c|c|c|c|}
\hline $\begin{array}{l}\text { MOBILITY } \\
\text { MEASURES }\end{array}$ & $\begin{array}{l}\text { VRB } \\
\text { GROUP }\end{array}$ & $\begin{array}{l}\text { MODEL } 1 \text { ESTIMATE } \\
(95 \% \mathrm{CI})\end{array}$ & $\mathbf{P}$ & $\begin{array}{l}\text { MODEL } 2 \text { ESTI- } \\
\text { MATE (95\% CI) }\end{array}$ & $\mathbf{P}$ & $\begin{array}{l}\text { MODEL } 3 \text { ESTI- } \\
\text { MATE (95\% CI) }\end{array}$ & $\mathbf{P}$ & $\begin{array}{l}\text { MODEL } 4 \text { ESTI- } \\
\text { MATE }(95 \% \text { CI })\end{array}$ & $\mathbf{P}$ \\
\hline \multirow[t]{5}{*}{ SPPB Total } & & & $0.01 *$ & & 0.49 & & 0.10 & & 0.05 \\
\hline & None & 1 [Reference] & & 1 [Reference] & & 1 [Reference] & & 1 [Reference] & \\
\hline & One & $0.32(-0.007,0.641)$ & 0.05 & $0.05(-0.257,0.362)$ & $0.74 \dagger$ & $0.20(-0.139,-0.013)$ & $0.23 \dagger$ & $0.22(-0.101,0.545)$ & $0.18 \dagger$ \\
\hline & Two & $0.20(-0.164,0.554)$ & 0.29 & $0.16(-0.183,0.498)$ & $0.36 \dagger$ & $0.26(-0.080,0.632)$ & 0.13 & $0.12(-0.232,0.478)$ & $0.50 \dagger$ \\
\hline & Three & $-0.85(-1.367,-0.342)$ & 0.001 & $-0.38(-0.876,0.120)$ & $0.14 \dagger$ & $-0.63(-1.148,-0.116)$ & $0.02 \dagger$ & $-0.71(-1.223,-0.196)$ & $0.01 \dagger$ \\
\hline \multirow[t]{5}{*}{ Gait Speed } & & & $0.0003 *$ & & $0.04 *$ & & $0.03 *$ & & $0.004 *$ \\
\hline & None & 1 [Reference] & & 1 [Reference] & & 1 [Reference] & & 1 [Reference] & \\
\hline & One & $0.03(-0.003,0.055)$ & 0.08 & $0.008(-0.022,0.037)$ & $0.61 \dagger$ & $0.009(-0.020,0,038)$ & $0.54 \dagger$ & $0.01(-0.018,0.039)$ & $0.47 \dagger$ \\
\hline & Two & $0.005(-0.027,0.038)$ & 0.74 & $0.005(-0.028,0.037)$ & 0.76 & $0.017(-0.014,0.048)$ & 0.29 & $0.004(-0.028,0.036)$ & $0.80 \dagger$ \\
\hline & Three & $-0.09(-0.138,-0.046)$ & $<0.001$ & $-0.06(-0.107,-0.011)$ & $0.02 \dagger$ & $-0.065(-0.111,-0.019)$ & $0.005 \dagger$ & $-0.07(-0.118,-0.026)$ & $0.002 \dagger$ \\
\hline \multirow[t]{5}{*}{ Basic LLFI } & & & $0.003 *$ & & 0.07 & & $0.02 *$ & & $0.005^{*}$ \\
\hline & None & 1 [Reference] & & 1 [Reference] & & 1 [Reference] & & 1 [Reference] & \\
\hline & One & $1.74(-0.035,3.511)$ & 0.05 & $0.77(-1.013,2.552)$ & $0.40 \dagger$ & $1.05(-0.738,2.844)$ & $0.25 \dagger$ & $1.62(-0.248,3.487)$ & 0.09 \\
\hline & Two & $1.77(-0.193,3.734)$ & 0.07 & $2.07(0.110,4.030)$ & 0.04 & $2.29(0.338,4.233)$ & 0.02 & $2.16(0.100,4.210)$ & 0.04 \\
\hline & Three & $-5.36(-8.167,-2.562)$ & 0.0002 & $-3.57(-6.436,-0.695)$ & $0.02 \dagger$ & $-4.10(-6.922,-1.273)$ & $0.005 \dagger$ & $-5.37(-8.338,-2.399)$ & 0.0004 \\
\hline \multirow[t]{5}{*}{ Adv LLFI } & & & $<0.0001 *$ & & $0.004 *$ & & $0.003 *$ & & $<0.0001^{*}$ \\
\hline & None & 1 [Reference] & & 1 [Reference] & & 1 [Reference] & & 1 [Reference] & \\
\hline & One & $4.13(2.046,6.217)$ & 0.0001 & $2.35(0.396,4.309)$ & $0.02 \dagger$ & $2.71(0.666,4.762)$ & $0.01 \dagger$ & $3.57(1.423,5.723)$ & $0.001 \dagger$ \\
\hline & Two & $-0.39(-2.701,1.918)$ & 0.74 & $-0.52(-2.668,1.635)$ & 0.64 & $0.56(-1.670,2.785)$ & $0.62 \dagger$ & $-0.61(-2.973,1.758)$ & 0.614 \\
\hline & Three & $-8.24(-11.539,-4.946)$ & $<0.0001$ & $-4.85(-8.00,-1.702)$ & $0.003 \dagger$ & $-5.85(-9.083,-2.624)$ & $0.0004 \dagger$ & $-7.48(-10.896,-4.057)$ & $<0.0001$ \\
\hline
\end{tabular}

Model 1 adjusted for age, race, sex, PHQ-9, and MMSE Scores; Model $2=$ Model $1+$ leg strength; Model $3=$ Model $1+$ knee flexion range of motion (ROM); Model $4=$ Model $1+$ leg velocity; $(\dagger)=>10 \%$ change in estimate from Model representing significant mediation. 


\section{MOBILITY AND NEUROMUSCULAR ATTRIBUTES}

20-84\% for SPPB, 33-73\% for gait speed, 33-56\% for Basic LLFI, and $41-43 \%$ for Advanced LLFI models.

When knee flexion ROM was added to the model (Model $3)$, VRB was no longer associated with SPPB $(\mathrm{p}=0.10)$. Additionally, VRB coefficients were attenuated by $26-38 \%$ for SPPB, $28-70 \%$ for gait speed, 24-40\% for Basic LLFI, and $29-243 \%$ for Advanced LLFI models.

Model 4 shows results for the inclusion of leg velocity in the model. VRB was no longer associated with SPPB $(\mathrm{p}=0.05)$ and VRB coefficients were attenuated by $17-40 \%$ for SPPB and $20-67 \%$ for gait speed. Additionally, the coefficient for VRB category ONE was attenuated by $14 \%$ when leg velocity was added to the Advanced LLFI model (Table 3).

\section{Discussion}

This analysis found that VRB status was negatively associated with mobility, such that greater vascular burden is linked to greater limitation on both performance-based and patient-reported measures of mobility. Additionally, VRB status was associated with higher impairment in leg strength, leg velocity, and knee ROM. We also found that these same neuromuscular attributes partially mediated the association between VRB status and mobility measures, independent of age, race, sex, PHQ-9, and MMSE scores.

Multi-morbidity of metabolic conditions puts individuals at a higher risk for developing mobility limitations (2). Our study demonstrated that VRB, as defined by the absence or presence of type 2 diabetes, hypertension, and obesity, was associated with worse performance on the SPPB, slower gait speed, and lower scores on the Basic and Advanced LLFI. Our findings are consistent with other research addressing mobility and metabolic syndrome as well as vascular risk factors for coronary heart disease and cardiovascular disease. For example, a study of metabolic syndrome by Penninx et al observed that older adults with metabolic syndrome had a $50 \%$ higher chance of developing future mobility limitations (16).

Another important finding from this study is that VRB status was linked to certain neuromuscular attributes. Individuals with the greatest VRB (i.e., three) had lower leg strength, compared to those with less burden. Additionally, there was a weak, yet significant association between VRB and knee flexion, such that those with two or three vascular risks had a lower degree of knee flexion ROM, compared to those with no or one vascular risk. Post hoc comparison of VRB status and leg velocity determined that those with three vascular risks manifested significantly slower leg velocity, compared to those with one vascular risk. Trunk muscle endurance may also have clinical relevance (11), but this association was difficult to detect given the small size of the Three VRB group and diversity of the vascular risks within each group. However, other studies have shown independent links between type 2 diabetes, obesity, or hypertension and neuromuscular consequences (17-19). For example, studies report that regardless of neuropathy, diabetic patients as well as those who are obese have decreased lower extremity muscle strength and power measures (2022). One longitudinal study found a $50 \%$ accelerated skeletal muscle decline in those with type 2 diabetes, compared to those without, while another study reported that obesity was linked to a four-fold higher incidence of developing knee osteoarthritis, which impairs knee $\operatorname{ROM}(20,23)$. These findings are supported by our results which suggest that lower leg strength, leg velocity, and knee flexion ROM is linked to the burden of underlying vascular disturbances.

The capacity to perform independent functioning depends upon the integrity of the varied body systems that underlie performance. In the current study, leg strength, leg velocity, and knee ROM were all linked to mobility skills among primary care patients with varying VRB status. The exact linkage between vascular/metabolic conditions and the manifestation of mobility limitations is not known. However, this association may be due to the vascular damage both centrally in the brain and in the periphery caused by type 2 diabetes, hypertension, and obesity. These vascular changes can manifest as reduced arterial wall compliance, arterial thickness and stiffening, endothelial dysfunction, and impaired relaxing and contracting mechanisms which leads to peripheral vascular resistance (24-26). These impairments also support studies associating vascular risk conditions with brain atrophy and reduced blood flow in the brain $(27,28)$. Moreover, our findings build upon prior research focusing upon mobility problems in general and among individuals with peripheral artery disease $(3,19,29)$. When taken together, vascular risk conditions cause inadequate supply of blood and oxygen levels to meet the metabolic demands associated with exercises as well as other physical activities.

Higher vascular risk burden is often associated with aging, lower cognitive functioning, higher BMI and mortality rates (30-32). Interestingly, MMSE scores were not able to differentiate VRB within this cohort. This could be due to the variation in group sample size, group differences in age, and/ or the protective factor of education. However, BMI was indeed able to differentiate the VRB groups, such that those with the highest VRB were those with the highest BMI. Within the current study, the group with the highest VRB was significantly younger than those with no VRB. This is could be due to the smaller sample of Three VRB compared to the None VRB groups. Additionally, the None group consisted of a wider age range $(66-91)$ compared to the Three VRB group $(65-83)$. We speculate that those with no VRB are living longer and ultimately are able to be studied, while those with higher VRBs, whom are at higher risk for mortality, are not.

Our study has several limitations. Due to its cross-sectional design, we could not determine causality. This was not a population-based sample, and data collection was confined to a specific single healthcare system within the study area, thus our findings may not be generalizable to a more racially or ethnically diverse population in a different region of the 


\section{THE JOURNAL OF FRAILTY \& AGING}

country. The vascular conditions utilized were self-reported and obesity was clinically assessed, as opposed to the more specific metabolic syndrome condition of abdominal obesity. Additionally, data on hyperlipidemia was not collected in the parent study (Boston RISE). Lastly, neuropathy, specific to type 2 diabetes, was not included in the data collection; however, sensory loss was assessed as a variable of interest for all participants. The strengths of this study include extensive data collection of neuromuscular impairment status and severity in a large sample of older adult primary care patients with mobility difficulty. Outcomes measures in the current reflect a cohort that may be frail (e.g., low SPPB scores). Although we did not specifically measure frailty, our findings are likely to apply to frail older adults as well. Furthermore, all study measures were well established, valid, and reliable among older adults (6) and all of the neuromuscular attributes we studied are clinically relevant and prioritized within rehabilitative care.

In conclusion, our results suggest that greater VRB is associated with impaired neuromuscular integrity, specifically leg strength and velocity and knee ROM, which is associated with the manifestation of mobility limitation in older adults. These findings underscore the relevance of peripheral neuromuscular attributes that underlie mobility limitations among patients with VRB and, therefore, should be considered in rehabilitative treatment.

Funding: This work is supported by the National Institutes of Health (R01 grant number AG032052-03 and K24 grant number HD070966-01) and the National Center for Research Resources (grant number UL1RR025758-01). Manuscript preparation was supported by the National Institutes of Health (K99 grant number AG051766) awarded to A.J.J.

Acknowledgements: The sponsors had no role in the design and conduct of the study; in the collection, analysis, and interpretation of data; in the preparation of the manuscript; or in the review or approval of the manuscript.

Conflict of interest: The authors report no disclosures.

Ethical standards: The procedures followed were in accordance with the ethical standards of the Institutional Review Board and with the Helsinki Declaration of 1975.

Open Access: This article is distributed under the terms of the Creative Commons Attribution 4.0 International License (http://creativecommons.org/licenses/by/4.0/), which permits use, duplication, adaptation, distribution and reproduction in any medium or format, as long as you give appropriate credit to the original author(s) and the source, provide a link to the Creative Commons license and indicate if changes were made.

\section{References}

1. Pérez-Zepeda MU, González-Chavero JG, Salinas-Martinez R, Gutiérrez-Robledo LM. Risk factors for slow gait speed: A nested case-control secondary analysis of the mexican health and aging study. J Frailty Aging. 2015;4(3):139-143. doi:10.14283/ jfa.2015.63.

2. Welmer AK, Angleman S, Rydwik E, Fratiglioni L, Qiu C. Association of cardiovascular burden with mobility limitation among elderly people: A populationbased study. PLoS One 2013;8(5):e65815. doi: 10.1371/journal.pone.0065815.

3. Bean JF, Latham NK, Holt N, et al. Which neuromuscular attributes are most associated with mobility among older primary care patients? Arch Phys Med Rehabil
2013:94(12):2381-2388 doi: 10.1016/j.apmr.2013.07.026

4. Yang EJ, Lim S, Lim JY, Kim KW, Jang HC, Paik NJ. Association between muscle strength and metabolic syndrome in older Korean men and women: The Korean Longitudinal Study on Health and Aging. Metabolism 2012;61(3):317-324. doi: 10.1016/j.metabol.2011.07.005.

5. Barnett K, Mercer SW, Norbury M, Watt G, Wyke S, Guthrie B. Epidemiology of multimorbidity and implications for health care, research, and medical education: a cross-sectional study. Lancet 2012;380(9836):37-43. 043. doi:10.1016/S0140 6736(12)60240-2.

6. Holt NE, Percac-Lima S, Kurlinski LA, et al. The boston rehabilitative impairment study of the elderly: A description of methods. Arch Phys Med Rehabil 2013;94(2):347-355. doi: 10.1016/j.apmr.2012.08.217

7. Sangha O, Stucki G, Liang MH, Fossel AH, Katz JN. The self-administered comorbidity questionnaire: A new method to assess comorbidity for clinical and health services research. Arthritis Rheum 2003;49(2):156-163. doi: 10.1002/art.10993.

8. Guralnik JM, Simonsick EM, Ferrucci L, et al. A short physical performance battery assessing lower extremity function: Association with self-reported disability and prediction of mortality and nursing home admission. J Gerontol 1994;49(2):M85-94. doi: 10.1093/geronj/49.2.M85

9. Haley SM, Jette AM, Coster WJ, et al. Late Life Function and Disability Instrument: II. Development and evaluation of the function component. J Gerontol A Biol Sci Med Sci 2002;57(4):M217-22.

10. Callahan D, Phillips E, Carabello R, Frontera WR, Fielding RA. Assessment of lower extremity muscle power in functionally-limited elders. Aging Clin Exp Res 2007;19(3):194-199.

11. Suri P, Kiely DK, Leveille SG, Frontera WR, Bean JF. Increased trunk extension endurance is associated with meaningful improvement in balance among older adults with mobility problems. Arch Phys Med Rehabil 2011;92(7):1038-1043. doi: 10.1016/j.apmr.2010.12.044

12. Watkins M, Riddle DL, Lamb R, Personius W. Reliability of goniometric measurements and visual estimate of knee range of motion obtained in a clinical setting. Phys Ther 1991;71(2):90-97.

13. Maldonado G, Greenland S. Simulation study of confounder-selection strategies. Am J Epidemiol 1993;138(11):923-936.

14. Baron RM, Kenny DA. The moderator-mediator variable distinction in social psychological research: conceptual, strategic, and statistical considerations. J Pers Soc Psychol 1986;51(6):1173-1182.

15. Kirn DR, Reid KF, Hau C, Phillips EM, Fielding RA. What is a clinically meaningfu improvement in leg-extensor power for mobility-limited older adults? J Gerontol A Biol Sci Med Sci 2016;71(5):632-636. doi: 10.1093/gerona/glv207.

16. Penninx BW, Nicklas BJ, Newman AB, et al. Metabolic syndrome and physical decline in older persons: results from the Health, Aging And Body Composition Study. J Gerontol A Biol Sci Med Sci 2009;64(1):96-102. doi: 10.1093/gerona/gln005.

17. Leenders M, Verdijk LB, van der Hoeven L, et al. Patients with type 2 diabetes show a greater decline in muscle mass, muscle strength, and functional capacity with aging. J Am Med Dir Assoc 2013;14(8):585-592. doi: 10.1016/j.jamda.2013.02.006

18. Jensen GL, Hsiao PY. Obesity in older adults: Relationship to functional limitation. Curr Opin Clin Nutr Metab Care 2010.13(1):46-51. doi: 10.1097/ MCO.0b013e32833309cf.

19. McDermott MM, Criqui MH, Greenland $\mathrm{P}$, et al. Leg strength in peripheral arterial disease: associations with disease severity and lower-extremity performance. J Vasc Surg 2004:39(3):523-530. doi: 10.1016/j.jvs.2003.08.038.

20. Park SW, Goodpaster BH, Strotmeyer ES, et al. Accelerated loss of skeletal muscle strength in older adults with type 2 diabetes: The health, aging, and body composition study. Diabetes Care 2007;30(6):1507-1512. doi: 10.2337/dc06-2537.

21. Ijzerman TH, Schaper NC, Melai T, Meijer K, Willems PJ, Savelberg HH. Lower extremity muscle strength is reduced in people with type 2 diabetes, with and without polyneuropathy, and is associated with impaired mobility and reduced quality of life Diabetes Res Clin Pract 2012;95(3):345-351. doi: 10.1016/j.diabres.2011.10.026.

22. Stenholm S, Alley D, Bandinelli S, et al. The effect of obesity combined with low muscle strength on decline in mobility in older persons: Results from the InCHIANTI Study. Int J Obes 2009;33(6):635-644. doi: 10.1038/ijo.2009.62.

23. Fehring TK, Odum SM, Griffin WL, Mason JB, McCoy TH. The obesity epidemic: Its effect on total joint arthroplasty. J Arthroplasty 2007;22(6):71-76. doi: 10.1016/j. arth.2007.04.014

24. Stapleton PA, James ME, Goodwill AG, Frisbee JC. Obesity and vascular dysfunction. Pathophysiology 2008;15(2):79-89. doi: 10.1016/j.pathophys.2008.04.007

25. Rigaud AS, Forette B. Hypertension in older adults. J Gerontol A Biol Sci Med Sci 2001. 56(4):M217-M225

26. Marso SP, Hiatt WR. Peripheral arterial disease in patients with diabetes. J Am Coll Cardiol 2006.47(5):921-929. doi: 10.1016/j.jacc.2005.09.065.

27. Last D, Alsop DC, Abduljalil AM, et al. Global and regional effects of type 2 diabetes on brain tissue volumes and cerebral vasoreactivity. Diabetes Care 2007;30(5):11931199. doi: $10.2337 / \mathrm{dc06}-2052$.

28. Beauchet $\mathrm{O}$, Celle $\mathrm{S}$, Roche $\mathrm{F}$, et al. Blood pressure levels and brain volume reduction: a systemic review and meta-analysis. J Hypertens 2013;31(8):1502-1516. doi: 10.1097/HJH.0b013e32836184b5.

29. Singh N, Liu K, Tian L, et al. Leg strength predicts mortality in men but not in women 


\section{MOBILITY AND NEUROMUSCULAR ATTRIBUTES}

with peripheral arterial disease. J Vasc Surg 2010;52(3):624-631. doi: 10.1016/j. jvs.2010.03.066.

30. Slagter SN, van Waateringe RP, van Beek AP, van der Klauw MM, Wolffenbutte BHR, van Vliet-Ostaptchouk JV. Sex, BMI, and age differences in metabolic syndrome: the Dutch Lifelines Cohort Study. Endocr Connect 2017;6(4):278-288. doi: 10.1530/EC-17-0011

31. Panza F, Frisardi V, Capurso C, et al. Metabolic syndrome and cognitive impairment: current epidemiology and possible underlying mechanisms. J Alzheimers Dis 2010;21(3):691-724. doi: 10.3233/JAD-2010-091669.

32. Mozaffarian D, Kamineni A, Prineas RJ, Siscovick DS. Metabolic syndrome and mortality in older adults: the Cardiovascular Health Study. Arch Intern Med 2008;168:969-978. doi: 10.1016/j.jvs.2010.03.066 